\title{
Mechanical properties of Graphene: Molecular dynamics simulations correlated to continuum based scaling laws
}

\author{
B. Javvaji ${ }^{\text {a }}$, P.R. Budarapu ${ }^{\text {b,* }}$, V.K. Sutrakar ${ }^{c}$, D. Roy Mahapatra ${ }^{\text {a }}$, M. Paggi ${ }^{\text {b }}$, G. Zi ${ }^{\text {d }}$, T. Rabczuk ${ }^{\mathrm{e}, \mathrm{f}, *}$ \\ ${ }^{a}$ Department of Aerospace Engineering, Indian Institute of Science, Bangalore 560012, India \\ ${ }^{\mathrm{b}}$ IMT School for Advanced Studies Lucca, Piazza San Francesco 19, 55100 Lucca, Italy \\ ${ }^{\mathrm{c}}$ Aeronautical Development Agency, Defence Research and Development Organization, Bangalore 560017, India \\ ${ }^{\mathrm{d}}$ School of Civil, Environmental and Architectural Engineering, Korea University, 136-701, Republic of Korea \\ e Division of Computational Mechanics, Ton Duc Thang University, Ho Chi Minh City, Viet Nam \\ ${ }^{\mathrm{f}}$ Faculty of Civil Engineering, Ton Duc Thang University, Ho Chi Minh City, Viet Nam
}

\section{A R T I C L E I N F O}

\section{Article history:}

Received 22 February 2016

Received in revised form 18 May 2016

Accepted 3 August 2016

Available online 17 September 2016

\section{Keywords:}

Graphene fracture

Molecular dynamics

Size-scale effects

Size effect law

Multifractal scaling law

Lattice orientation effect

\begin{abstract}
A B S T R A C T
In this paper, the combined effect of domain size, lattice orientation and crack length on the mechanical properties of Graphene, namely the yield strength and strain, are studied extensively based on molecular dynamics simulations. Numerical predictions are compared with the continuum-based laws of size effect and multifractal scaling. The yield strength is found to vary with the specimen size as $\approx L^{-1 / 3}$, which is in agreement with the multifractal scaling law, and with the inverse square of the initial crack length as $\approx a_{0}^{-1 / 2}$, according to the Griffith's energy criterion for fracture.
\end{abstract}

๑) 2016 Elsevier B.V. All rights reserved.

\section{Introduction}

Graphene is an extensively used material in a wide range of applications, where it appears to be very promising due to its unique properties, see $[33,11,57]$, and several useful material properties [26,39,61]. Because of its extreme mechanical properties [24], Graphene can be used as a strengthening component in composites [32,19]. As the demand for Graphene-based applications is growing, it is important to understand the mechanical properties that affect the device performance and reliability. The mechanical properties of bulk Graphene are extensively studied both based on experiments $[34,30]$ and simulations $[60,37]$. However, due to the practical difficulties in setting-up experiments [31], experimental characterization of the mechanical properties of Graphene is still challenging. Therefore, numerical simulations and multiscale methods for fracture [63-67] are good alternatives for predicting fracture related properties of Graphene. Tiwary et al. [51] have performed numerical simulations to come up with a chemical free

\footnotetext{
* Corresponding authors at: IMT School for Advanced Studies Lucca, Italy (P.R. Budarapu); Division of Computational Mechanics, Ton Duc Thang University, Ho Chi Minh City, Viet Nam (T. Rabczuk).

E-mail addresses: pattabhi.budarapu@imtlucca.it (P.R. Budarapu), timon.rabczuk @tdt.edu.vn (T. Rabczuk).
}

technique to synthesize Graphene. Several researchers have investigated the mechanical properties of Graphene based on molecular dynamics (MD) simulations $[35,28,25]$ and continuum based techniques, as explained below.

Zheng et al. [62] investigated the mechanical properties based on MD simulations, of Graphene nanochain which is constructed by sewing up pristine or twisted Graphene nanoribbons (GNRs) and interlocking the obtained nanorings. The effects of length, width and twist angle of the constituent GNRs on the mechanical performance were also analyzed. Yang et al. [55] performed MD simulations to study the deformation behavior of nanocrystalline Graphene sheets. They found that Graphene sheets have almost the same stress and strain at fracture, but a decreasing elastic modulus with grain size. Dewapriya et al. [23] have performed MD simulations to investigate the temperature and strain rate dependent fracture strength of defective Graphene. They also came up with a continuum based fracture mechanics framework to characterize the temperature and strain rate dependent strength of defective Graphene sheets [22].

Georgantzinos et al. [27] used an atomistic, spring-based, nonlinear finite element method to predict the mechanical behavior of Graphene nanoribbons. They reported that the linear and nonlinear mechanical properties are strongly dependent on the 
structure as well as on the size of the Graphene strip. A micromechanical finite element approach was proposed in [45], to estimate the elastic mechanical properties of Graphene reinforced composites. They studied the composite materials consisting of Graphene monolayers uniformly distributed within the matrix, by considering a representative volume element. Liu et al. [36] studied the mechanical properties of various interlayer and intralayer cross links through first-principles calculations, and performed continuum based analysis for the overall mechanical properties of Graphene-based paper materials. They established a characteristic length $\left(l_{0}\right)$ such that when the size of the Graphene sheets exceeds $3 l_{0}$, the tension-shear (TS) chain model fails to predict the overall mechanical properties of the Graphene-based papers.

Some size based studies reported in the literature on the estimation of the mechanical properties of Graphene are as follows. Yin et al. [56] demonstrated that the Griffith criterion is valid for small cracks of lengths up to $10 \mathrm{~nm}$, where the strength of shorter cracks is over estimated. Sun et al. [47] have shown that the yield strength of both zigzag and armchair Graphene nanoribbons decreases with the ribbon length changing from $240 \AA$ to $30 \AA$. However, the ductility of armchair Graphene nanoribbons was found to be improved with size reduction. Xu et al. [54] investigated the size-dependent mechanical properties and the edge effect of the tangential interface between Graphene and a polyethylene terephthalate substrate (PET) by in-situ Raman spectroscopy experiments. They observed the existence of the edge effect in the interfacial stress/strain transfer process, and the length of the edge of the interface can be affected by the size of Graphene. Wang et al. [53] studied the elastic properties of monolayer and multilayer Graphene nanofilms based on a structural mechanics approach. They proved the estimated Young's moduli and Poisson's ratios of monolayer Graphene nanofilms are size and chirality-dependent. They also validated the predicted Young's moduli and Poisson's ratios with the experimental results. Chu et al. [20] have investigated the size effect of finite-sized Graphene nanoribbons under uniaxial tension, based on MD simulations using an adaptive intermolecular reactive empirical bond order (AIREBO) potential. They reported the size effect and aspect ratio effects are significant in zigzag nanoribbons compared to the armchair nanoribbons.

Zhang et al. [58] reviewed the recent progress in experimental and theoretical studies on the fracture behaviors of Graphene. They reported that there are still significant yet unresolved issues related to the fracture of Graphene, in the areas of: size effect on the strength of polycrystalline Graphene; design of the Graphene structures with controlled fracture; influence of out-of-plane effects on fracture of Graphene; continuum modeling of Graphene fracture to capture the complicated modes, such as shear fracture coupled with wrinkling deformation and tear fracture, control the fracture behavior of Graphene by combing the chemical, irradiation and stress effects, to name a few. Datta et al. [21] have investigated the effect of different crack lengths in mixed mode loading conditions at $300 \mathrm{~K}$, in the arm-chair and zig-zag Graphene by aligning the crack perpendicular to the lattice orientation. They also studied the effect of crack orientation in the arm-chair and zig-zag Graphene, retaining the same loading directions. Budarapu et al. [10] have studied the crack initiation and growth mechanisms in Graphene based on MD simulations. They studied the influence of the time step and the temperature on the post yielding behavior of Graphene based on a systematic study of the bond stretching and bond reorientation phenomena.

However, the combined effect of domain size and lattice orientation of initially notched Graphene sheets on its apparent mechanical properties is yet to be investigated in detail. Therefore, in the present work, an attempt has been made to derive the relation between the domain size, lattice orientation and the initial crack length on the mechanical properties of Graphene. Results based on molecular dynamics simulations are correlated to the existing continuum based scaling laws. The arrangement of the article is as follows: details of the numerical model are explained in Section 2. Details of the continuum based scaling laws and their correlations to MD simulations are discussed in Section 3. The effect of domain size, lattice orientation and crack length on the yield properties of Graphene are explained in Section 4. Key findings are summarized in Section 5.

\section{Modelling and simulations}

The main objective of the present work is to understand the combined effect of domain size, lattice orientation and the initial crack length on the mechanical properties of Graphene. To achieve the objective, uniaxial tensile tests on Graphene sheets are performed by varying domain size $(\mathrm{L})$, lattice orientation angle $(\theta)$ and initial crack length $\left(a_{0}\right)$.

The atomistic model in the present work is developed by considering the hexagonal lattice structure of the Graphene sheet with a lattice constant $2.49 \AA$, see Fig. 1(a). The atom to atom interactions of carbon in Graphene are modeled based on the Tersoff potential [49], which has been successfully applied to predict mechanical properties of Graphene $[9,52,50,10,68]$. Variation of the fracture strength with the cut-off distance $\left(r_{c}\right)$ at $0 \mathrm{~K}$ is plotted in Fig. 2. According to Fig. 2 and to [44,59], a cut-off distance of $2.1 \AA$ is considered to reproduce the physical observations. Hence, $r_{c}=2.1 \AA$ is used in all the simulations of the present work. An initial edge crack is created parallel to the $x$ direction, in the middle of the domain along the $y$ direction, as shown in Fig. 1(b). The corresponding full scale atomistic model consists of a total number of atoms ranging from 3678 to 299936, for the domain sizes of $10 \mathrm{~nm}$ to $86 \mathrm{~nm}$, depending on the lattice orientation. The initial crack is modeled by restricting the interactions between the atoms on either side of the crack surface, which is achieved by updating the neighbor list accordingly. Crack propagation is studied based on the natural bond breaking. The degrees of freedom along the $x$ direction of the left and right edge atoms and along the $y$ direction of the top and bottom edge atoms are restrained. An initial velocity of 0.1 angstroms/ pico-seconds ( $\AA / \mathrm{ps})$ along the $y$ direction is prescribed on the top and bottom edge atoms to study the crack growth.

In this work, the load is applied in terms of 'load cycles', which is defined as prescribing the velocity on the top and bottom edge atoms for a specified time period, followed by an equilibration for another specified time period. In each load cycle, the prescribed velocity on the top and bottom edge atoms is applied for a period of $1 \mathrm{ps}$, after which the system is equilibrated for a period of $1 \mathrm{ps}$. The stress computed in the present work is the averaged stress estimated based on the Virial theorem $[38,46]$. The average virial stress $(\boldsymbol{\sigma})$ over a volume $\Omega$ with total number of atoms $n^{A}$ is calculated as

$$
\boldsymbol{\sigma}=\frac{1}{\Omega}\left(\frac{1}{2} \sum_{\substack{\alpha=1 \\ n^{A}}}^{n^{n^{A}}, 1,} \mathbf{r}_{\alpha \beta} \otimes \mathbf{f}_{\alpha \beta}-\sum_{\alpha=1}^{n^{A}} m_{\alpha} \dot{\mathbf{u}}_{\alpha} \otimes \dot{\mathbf{u}}_{\alpha}\right)
$$

where $m_{\alpha}$ is the mass of atom $\alpha, \mathbf{r}$ is the position vector, $\mathbf{f}$ is the force vector and $\mathbf{u}_{\alpha}, \dot{\mathbf{u}}_{\alpha}$ are the displacement and velocity vectors of atom $\alpha$, respectively. Engineering strain is used as a measure of deformation, which is defined as $\left(l-l_{0}\right) / l_{0}$, where $l$ is the instantaneous length and $l_{0}$ is the initial length of Graphene obtained after the first step of energy minimization corresponding to the initial configuration. The nominals strength in this work is extracted at 


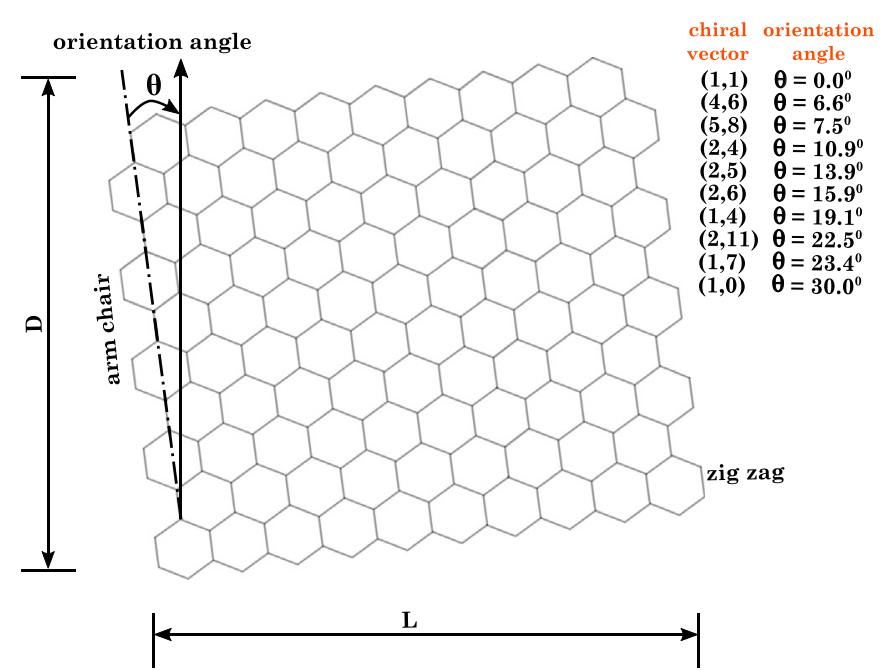

(a)

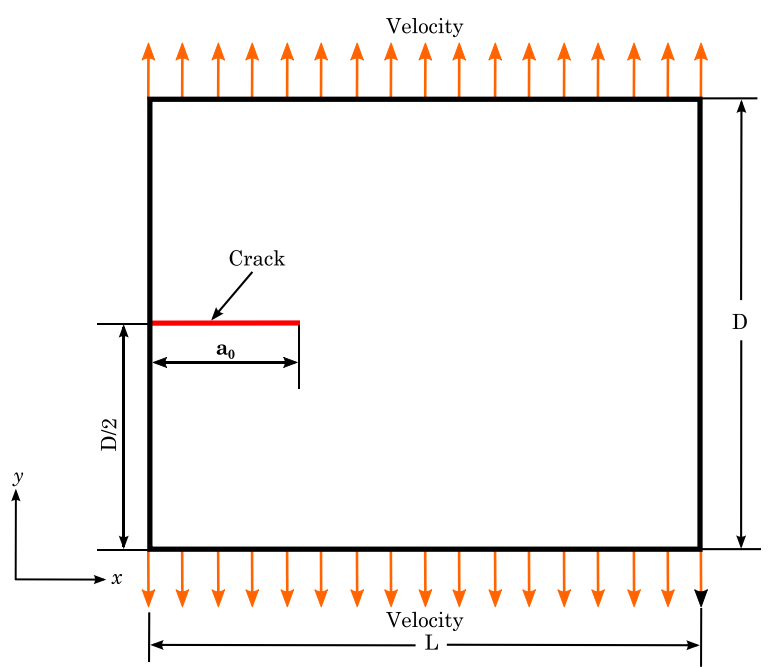

(b)

Fig. 1. Schematic of the (a) graphene lattice showing the orientation angles and the (b) atomistic model used in the simulations.

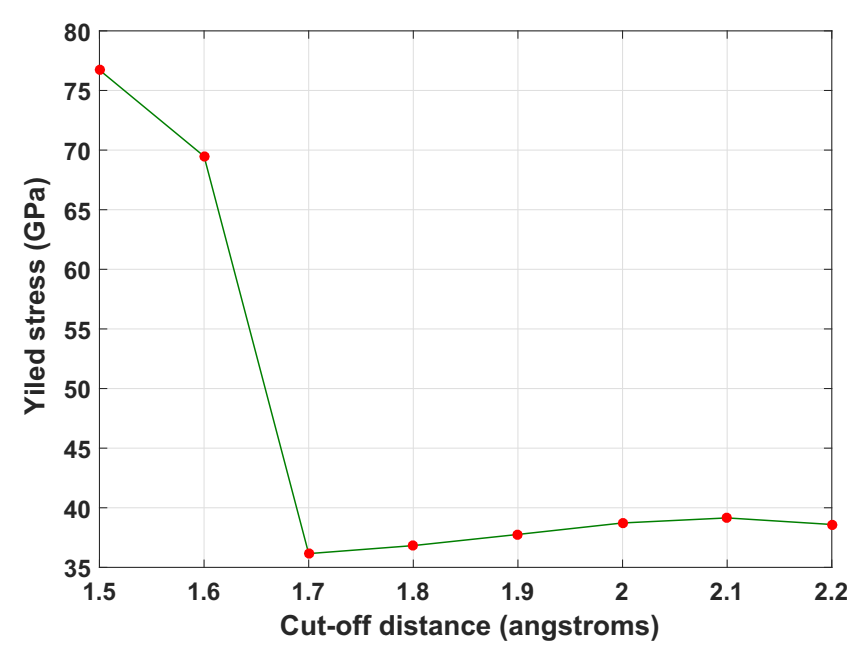

Fig. 2. Variation of the yield stress as a function of the cut-off distance.

the time of first bond break from the stress-strain curve. The yield strain is estimated as the strain at the first bond break from the strain-time data. The equations of motion and the time integration is carried out based on the Verlet algorithm [48]. The effect of numerical stability has been investigated in [10]. Budarapu et al. [10] have reported that $1.0 \mathrm{fs}$ is sufficient to study the mechanical behavior of Graphene up to yielding. However, a much smaller time step is required to predict the crack growth more accurately. Since the objective is to study the yield properties, a time step of $1.0 \mathrm{fs}$ has been considered in the present work.

All the simulations are performed under isothermal loading conditions, at a temperature of $\approx 0 \mathrm{~K}(0.1 \mathrm{~K})$. In the isothermal loading, the system temperature is maintained constant. However, the application of the initial velocities would lead to increase in the kinetic energy and hence, in the system temperature. Therefore, the isothermal conditions in the present work are achieved by velocity rescaling technique, where the velocities at each time step are rescaled to maintain the constant temperature of $\approx 0 \mathrm{~K}$. The temperature of $\approx 0 \mathrm{~K}$ is considered to avoid the influence of temperature on the lattice orientations and crack length in the mechanical behavior of Graphene.

In this paper, we performed simulations by considering six different square domains (see Fig. 1(b)) of dimensions of $10 \times 10 \mathrm{~nm}^{2}$,
$20 \times 20 \mathrm{~nm}^{2}, \quad 30 \times 30 \mathrm{~nm}^{2}, \quad 43 \times 43 \mathrm{~nm}^{2}, \quad 60 \times 60 \mathrm{~nm}^{2}, \quad$ and $86 \times 86 \mathrm{~nm}^{2}$, with three different lattice orientations indicated by chiral vectors: $(1,1),(2,5)$ and $(1,0)$, with corresponding lattice orientation angles of $0^{\circ}, 13.9^{\circ}$ and $30^{\circ}$, and thirteen different initial crack lengths equal to: $0.025 L, 0.05 L, 0.1 L, 0.15 L, 0.2 L, 0.25 L$, $0.33 L, 0.4 L, 0.5 L, 0.6 L, 0.7 L, 0.8 L$, and $0.9 L$, where $L$ is the width of the sample, as shown in Fig. 1. Therefore, in total 252 simulations are carried out by varying the domain size, lattice orientation and crack length, including the simulations without crack. All the simulations in the this work are carried out using the open source Large-scale Atomic/Molecular Massively Parallel Simulator (LAMMPS) software [43].

\section{Continuum based scaling laws}

In this work, results based on the molecular dynamics simulations are correlated to the size effect law (SEL) proposed by Bažant et al. [5] and to the multifractal scaling law (MFSL) proposed by Carpinteri [13], used in continuum mechanics.

The original size effect law by Bažant et al. [5] was further improved in $[2,4,3]$, based on the information from larger specimen sizes. The established size effect law in Bažant et al. [5] is mainly governed by the total dissipated energy due to fracture $\left(W_{f}\right)$. According to [5], when a quasi-brittle structure has a deep notch or a large traction free crack that has formed before reaching the maximum load, the size effect on the mean nominal strength of structure is essentially energetic, with a negligible statistical effect $[6,8]$. This is type II size effect law for the problems with a large crack, which is described in [1,5] and is given by:

$\boldsymbol{\sigma}_{N}=B \boldsymbol{\sigma}_{y}\left(1+\frac{L}{L_{0}}\right)^{-1 / 2}$,

where $\boldsymbol{\sigma}_{N}$ is the nominal stress at failure, $\boldsymbol{\sigma}_{y}$ is the uni-axial yield strength, $L_{0}$ is the transitional size which depends on the characteristic length and the geometry and $B$ is the empirical constants which depend on the geometrical shape of the structure but not on the size of the domain. Based on Eq. (2), the nominal strength is inversely dependent on the domain size, viz. $1 / \sqrt{L}$.

On the other hand, Carpinteri [12,13] proposed to model concrete damage by assuming that the rarefied resisting crosssections in correspondence to the peak load, $A_{\text {res }}^{*}$, can be represented by stochastic lacunar fractal sets with dimension 


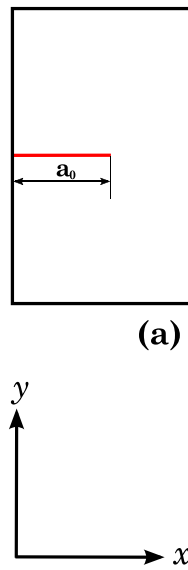

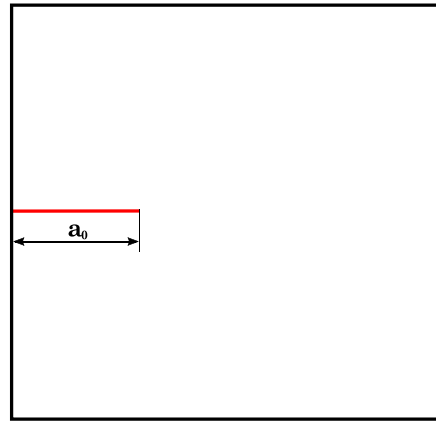

(b)

$a_{0} / D=$ constant

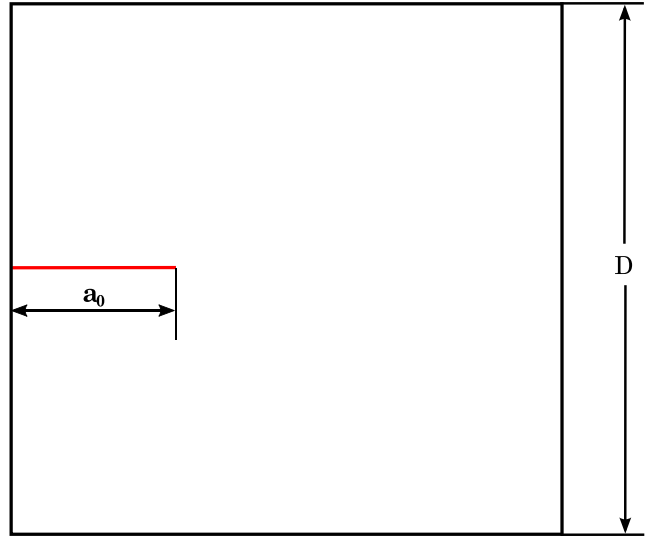

(c)

Fig. 3. Schematics of the domains having same aspect ratios, used in the simulations.
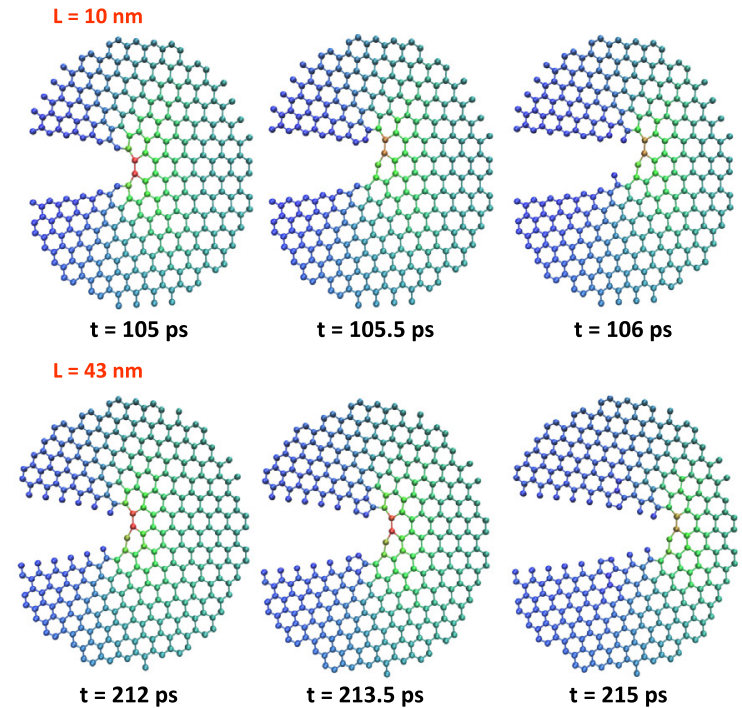

$\mathrm{L}=60 \mathrm{~nm}$

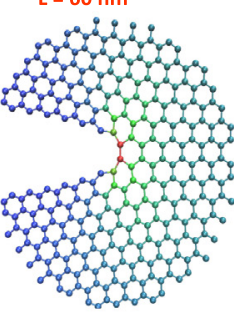

$\mathbf{t}=\mathbf{2 6 1}$ ps

$\mathrm{L}=86 \mathrm{~nm}$

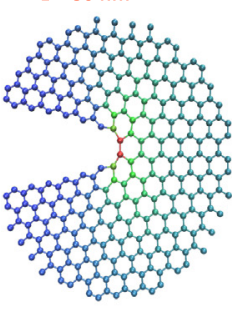

$\mathrm{t}=319.5 \mathrm{ps}$

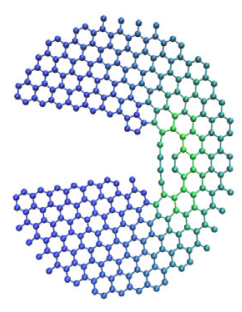

$t=261.5$ ps

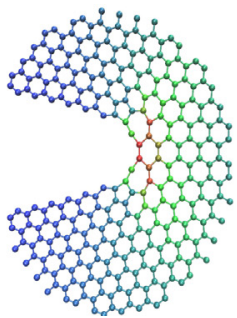

$=\mathbf{3 2 0}$ ps
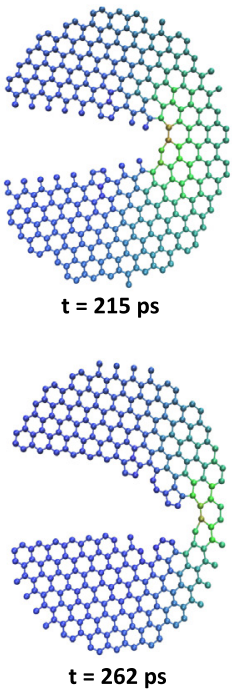
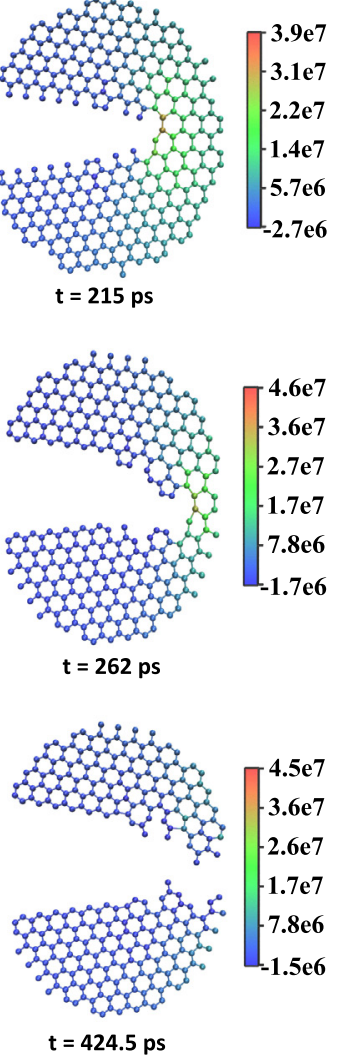

(a) Lattice oriented along $0^{\circ}$
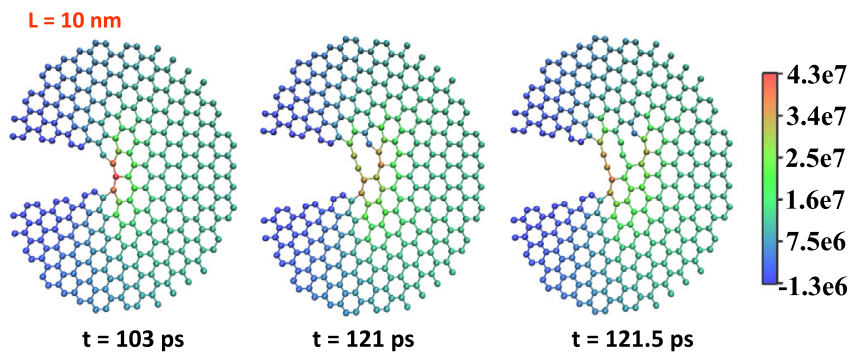

$\mathrm{L}=43 \mathrm{~nm}$

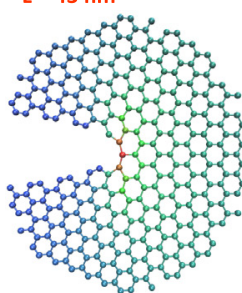

$t=216$ ps
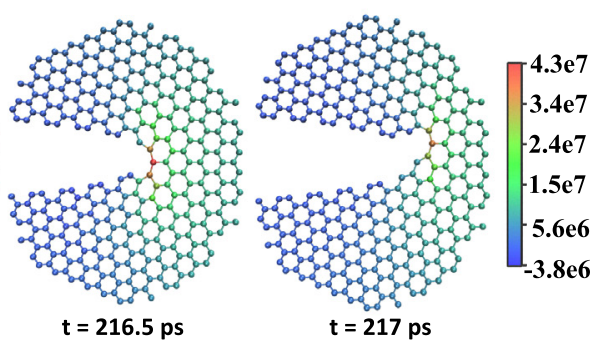

$\mathrm{L}=60 \mathrm{~nm}$
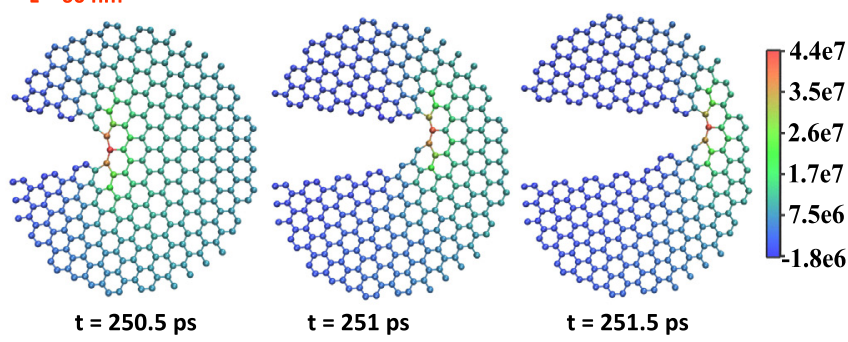

$\mathrm{L}=86 \mathrm{~nm}$
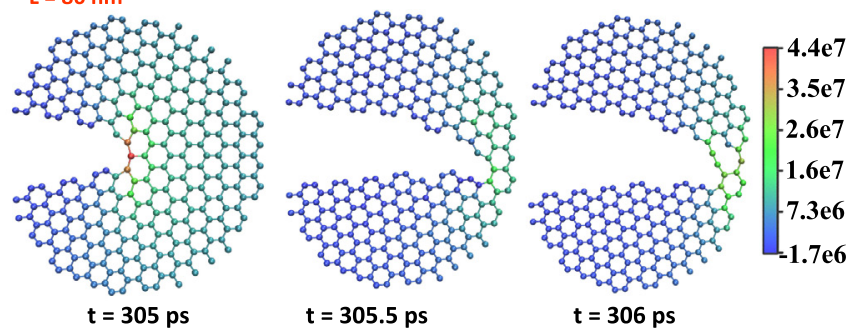

(b) Lattice oriented along $30^{\circ}$

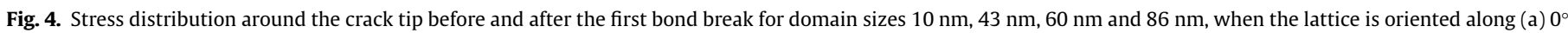
and (b) $30^{\circ}$. Configurations just before and after the first bond break and after the second bond break, are shown in the first, second and third columns, respectively. 
$2-d_{\sigma}\left(d_{\sigma} \geqslant 0\right)$. Based on this assumption, the following scaling law for the nominal tensile strength was derived:

$\sigma_{N}=\sigma^{*} L^{-d_{\sigma}}$

where $\sigma^{*}$ is a scale invariant mechanical property, see also $[14,16,41]$, with anomalous physical dimensions $[F][L]^{2-d_{\sigma}}$. Eq. (3) represents a power law with negative exponent $-d_{\sigma}$, indicating the negative size effect on the tensile strength, as discussed above. However, the single fractal scaling is valid for a size-limited scale range. As the size increases, fractality slowly vanishes $\left(d_{\sigma} \rightarrow 0\right)$ with a corresponding homogenization of the failure domain $[18,17]$. Therefore, the mechanical properties can be assumed to depend on the ratio between a characteristic material length $\left(l_{c h}\right)$ and the size of the specimen $(L)$. Carpinteri [13] reported that the experimental values of $d_{\sigma}$ always lay between 0 and $1 / 2$. For extremely brittle and disordered materials, the dimensional decrement $d_{\sigma}$ tends to the Linear Elastic Fracture Mechanics (LEFM) limit, 1/2. Based on this, Carpinteri [13] and Carpinteri et al. [15] have proposed the multifractal scaling law for tensile strength as:

$\boldsymbol{\sigma}_{N}=f_{t}\left(1+\frac{l_{c h}}{L}\right)^{1 / 2}$

The scaling law in Eq. (4) is considered as a two-parameter model, where the asymptotic value of the nominal quantity, $f_{t}$, corresponds to the lowest nominal tensile strength and it is reached in the limit of infinite sizes. The salient features and differences with applicability of SEL and MSFL were addressed in [7]. In the present work, an attempt has been made to correlate the MD results from various

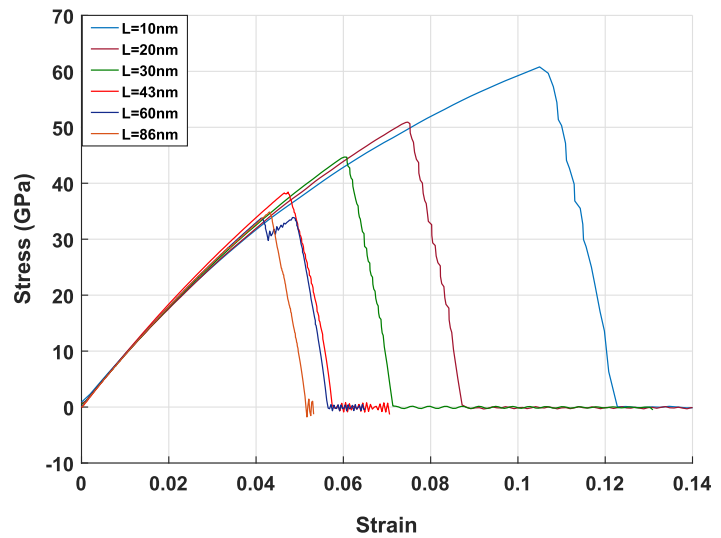

(a) Lattice oriented along $0^{\circ}, \mathrm{a}_{0}=0.5 \mathrm{~L}$

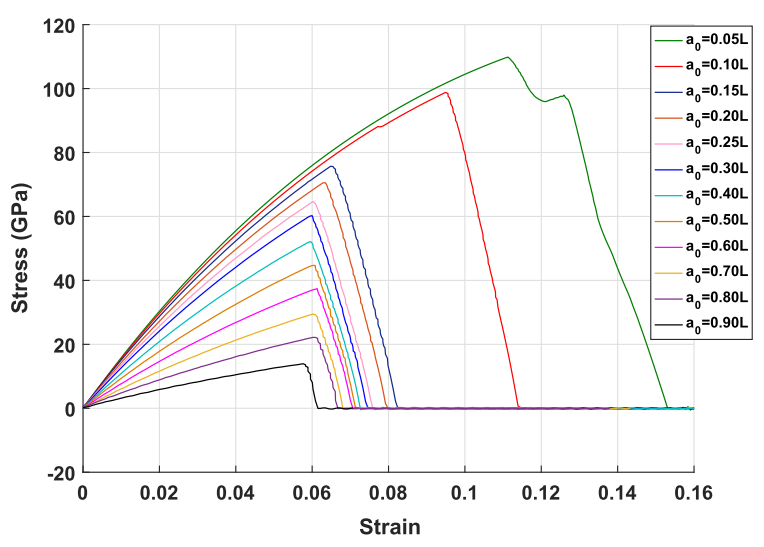

(c) Lattice oriented along $0^{\circ}, \mathrm{L}=30 \mathrm{~nm}$ size and lattice orientations of Graphene based on the MD simulations to SEL and MFSL.

\section{Results and discussion}

In this work, we considered domains of similar shapes with different sizes to maintain the same aspect ratio as shown in Fig. 3. Fig. 4 shows a closeup of the stress distribution on the deformed lattice configuration around the crack tip just before and after the breakup of first few bonds for domain sizes of $10 \mathrm{~nm}, 43 \mathrm{~nm}$, $60 \mathrm{~nm}$ and $86 \mathrm{~nm}$, when the lattice is oriented along $0^{\circ}$ and $30^{\circ}$ in Fig. 4(a) and (b), respectively. In the initial configuration, all the atoms are assumed to have the same potential energy. From Fig. 4, the fracture pattern is observed to be changing with the domain size and lattice orientation. Moreover, as the domain size increases, the time for the first bond break increases and the subsequent crack growth after the first bond break is observed to be fast. This is because as the domain size increases, the distance between the crack surface to the boundaries also increases. For example, the distance between crack surface and the top surface is $4.5 \mathrm{~nm}$ and $45 \mathrm{~nm}$ for domains sizes of $10 \mathrm{~nm}$ and $86 \mathrm{~nm}$, respectively. This will result in a very fast load transfer from the top surface to the crack surface, for small domain sizes. Furthermore, the number of bonds broken after the first bond break are observed to be increasing with domain size for all the lattice orientations. This is because, increase of the specimen size increases the number of relatively weak bonds, which offers more possibilities for bond breaking and dislocations to occur, and thus reducing the strength

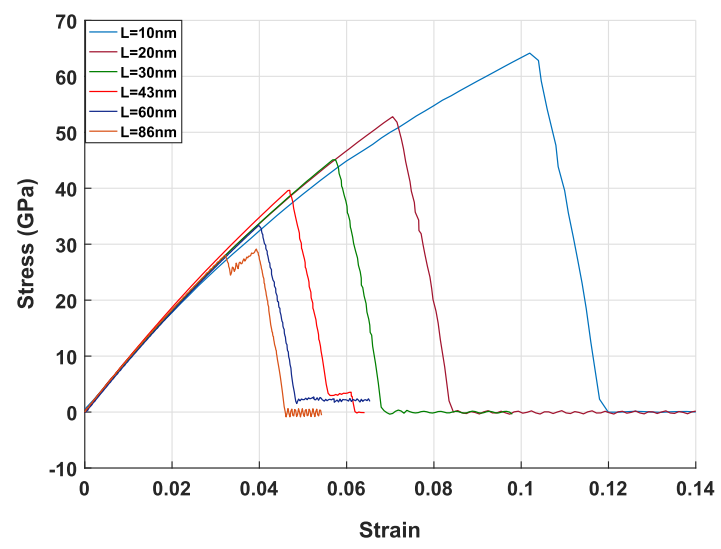

(b) Lattice oriented along $30^{\circ}, \mathrm{a}_{0}=0.5 \mathrm{~L}$

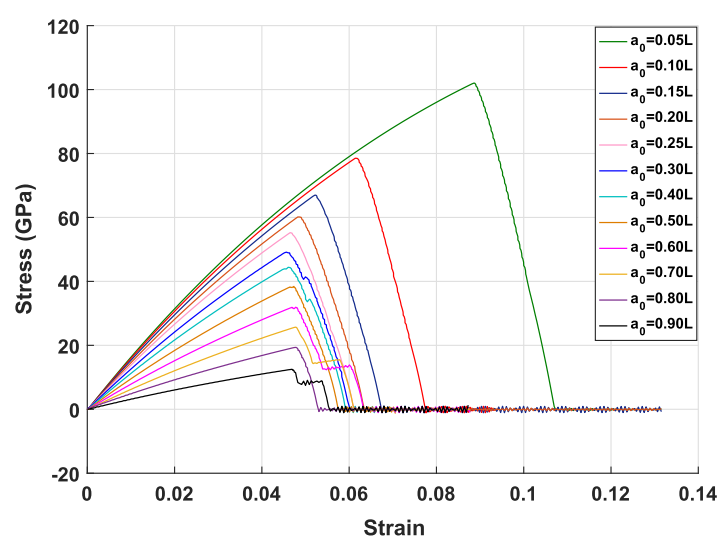

(d) Lattice oriented along $0^{\circ}, \mathrm{L}=43 \mathrm{~nm}$

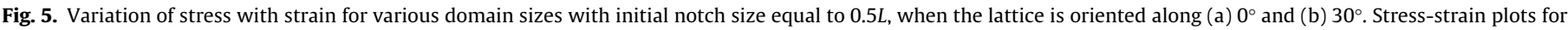
domain sizes of (c) $30 \mathrm{~nm}$ and (d) $43 \mathrm{~nm}$, for different initial notch sizes. 
of the specimen. Therefore, the mechanical stiffness of small domains is higher as compared to that typical of large domains. As a result, the yield values at the time of first bond break are higher for small domains, which can be noticed from Fig. 5(a) and (b) when the lattice is oriented along $0^{\circ}$ and $30^{\circ}$, respectively.

Distribution of the stress with strain for various domain sizes, lattice orientations and initial notch sizes are plotted in Fig. 5. Stress-strain plots with different domain sizes with an initial notch size of $0.5 \mathrm{~L}$ when the lattice is oriented along $0^{\circ}$ and $30^{\circ}$ are plotted in Fig. 5(a) and (b), respectively. From Fig. 5(a) and (b), the yield strength can be observed to be decreasing with an increase in the domain size. This is mainly due to the fact that larger specimens offer more spaces for dislocations to initiate. Though constraints do exist in the form of boundary conditions in MD simulations, Graphene lattice is a discrete system with atoms distributed at certain positions. Atomic bonds are relatively weak parts compared to the individual atoms in the atomistic model. Therefore, increase of the specimen size increases the number of relatively weak bonds, which in turn offers more possibilities for bond breaking and dislocations to occur, and thus reduces the strength of the specimen. Stress-strain plots for different initial notch sizes when the lattice is oriented along $0^{\circ}$, for domain sizes of $30 \mathrm{~nm}$ and $43 \mathrm{~nm}$ are plotted in Fig. 5(c) and (d), respectively. From Fig. 5(c) and (d), the drop in yield strength is observed to be significant for initial notch sizes less than $0.2 \mathrm{~L}$. This is due to the fact that when the initial notch size is less than $0.2 L$, higher energy is required to initiate the crack growth, which leads to higher yield stress and strain, compared the case with initial notch size more than $0.2 L$.

Fig. 6 shows the distribution of the logarithm of yield stress $\left(\sigma_{N}\right)$ with the logarithm of the domain size $(L)$, when the lattice is oriented along the $0^{\circ}, 13.9^{\circ}$ and $30^{\circ}$ angles. The dotted lines in Fig. 6 represent the linear fits and the corresponding fitting equations are mentioned on top of the concerned curve. From Fig. 6, the slope of the linear fit for the unnotched specimen is found to be $\approx 0$, for all the domain sizes and lattice orientations. This confirms the fact that failure of unnotched materials obeys classical strength criteria irrespective of the type of analysis [1].

Based on LEFM, yield strength varies inversely as the square root of the domain size $(1 / \sqrt{L})$, as explained in Eq. (2). Therefore, according to LEFM, the slope of the $\log \left(\sigma_{N}\right)$ with $\log (L)$ plot should be $-1 / 2$. However, the average of the slopes of $\log \left(\sigma_{N}\right)$ vs. $\log (L)$ curves in Fig. 6, for notched specimens is estimated as $-0.334,-0.316$ and -0.344 , when the lattice is oriented along the $0^{\circ}, 13.9^{\circ}$ and $30^{\circ}$, respectively. This is in agreement with the experimental observations reported by $[13,18]$, where $d_{\sigma}$ is observed to vary between [0, $1 / 2$ ], as discussed in Section 3. This effect is presumably induced by the roughness of the crack path which is caused by the lattice geometry. The maximum variation in the average slope is $8.86 \%$. Hence, the effect of lattice orientation on the yield strength is small. Therefore, based on the present MD studies, the nominal yield strength $\left(\sigma_{N}\right)$ of Graphene are found to vary as $\approx \mathrm{L}^{-1 / 3}$.

According to Griffith's theory [29], brittle fracture occurs when the released strain energy exceeds the surface energy required for an infinitesimal extension of the crack. For an edge crack of initial length $a_{0}$, based on the Griffith criterion the critical stress at the onset of fracture, $\sigma_{N}$, can be expressed as a function of $a_{0}$, as [42]:

$\sigma_{N}=\sqrt{\frac{2 \gamma E}{\pi a_{0}}}$

where $E$ is Young's modulus and $\gamma$ is the surface energy, which is the edge energy for a 2D material like Graphene. Eq. (5) can be rearranged as,
$\sigma_{N} \sqrt{a_{0}}=\sqrt{\frac{2 \gamma E}{\pi}}$

The left-hand side of Eq. (6) contains only the computed fracture quantities, namely fracture stress and crack size, whereas the right-hand side depends only on material parameters. Therefore, based on Eq. (6), the Griffith theory of brittle fracture is applicable to Graphene, if the estimated pairs of $\sigma_{N}$ and $a_{0}$ results in a constant

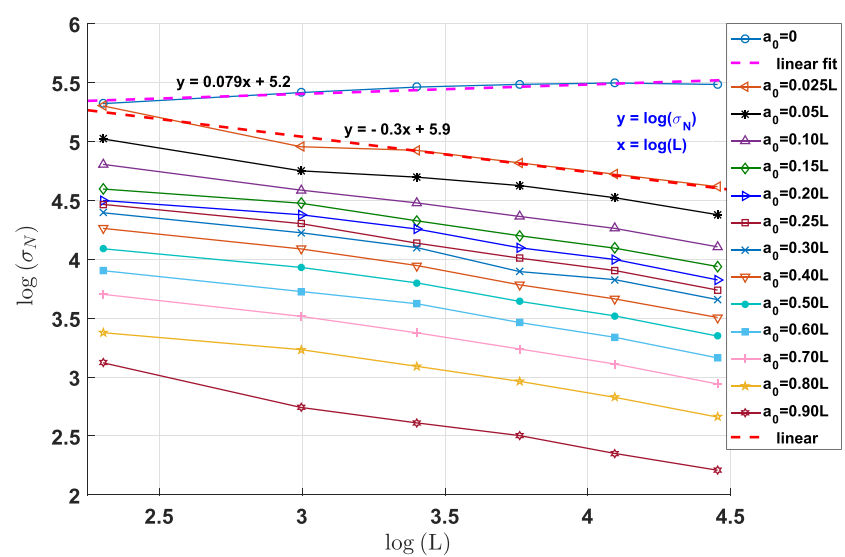

(a) Lattice oriented along $0^{\circ}$

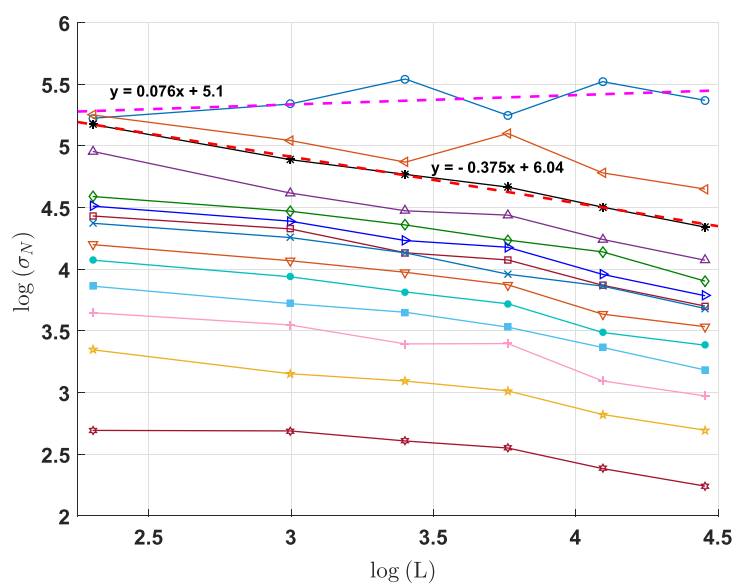

(b) lattice oriented along $13.9^{\circ}$

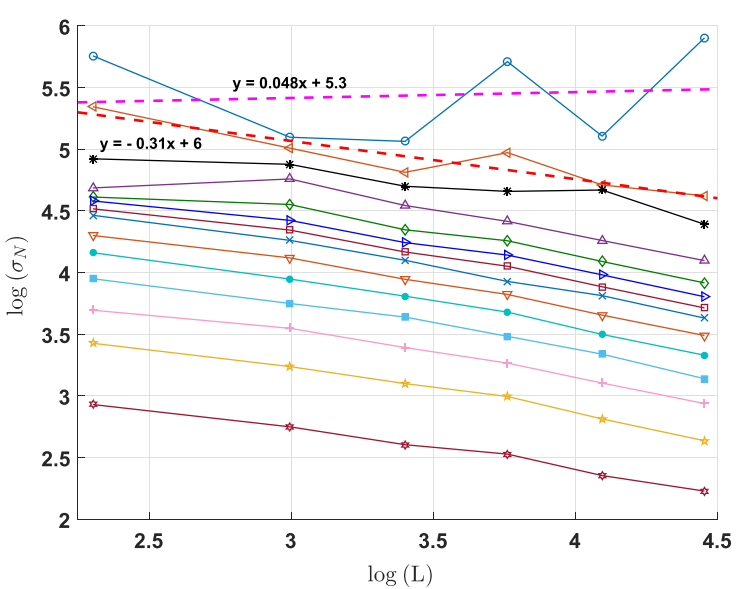

(c) lattice oriented along $30^{\circ}$

Fig. 6. Variation of the yield stress logarithm of $\left(\sigma_{y}\right)$ with the logarithm of domain size $(L)$, for different crack lengths, when the lattice is oriented along (a) $0^{\circ}$, (b) $13.9^{\circ}$ and (c) $30^{\circ}$, respectively. The dotted lines indicate the linear fits, corresponding fitted equations are mentioned on top of the concerned curve. 


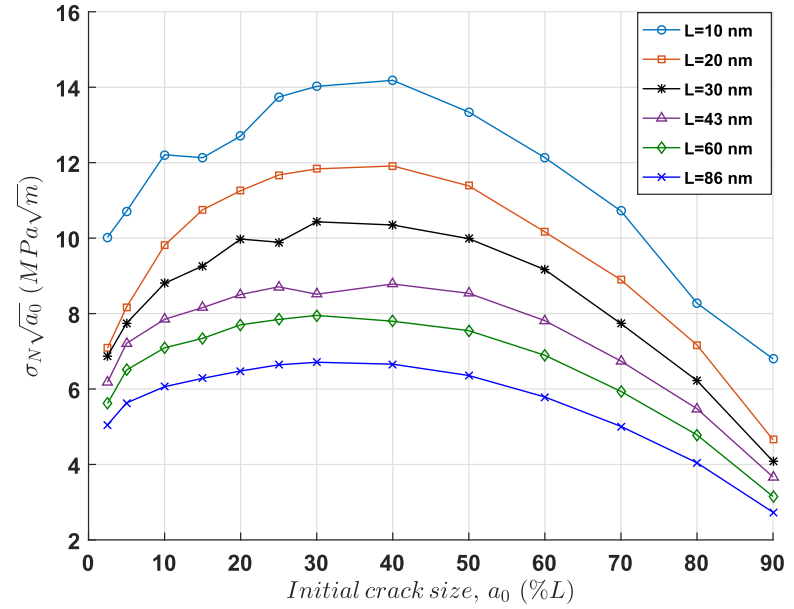

(a) Lattice oriented along $0^{\circ}$

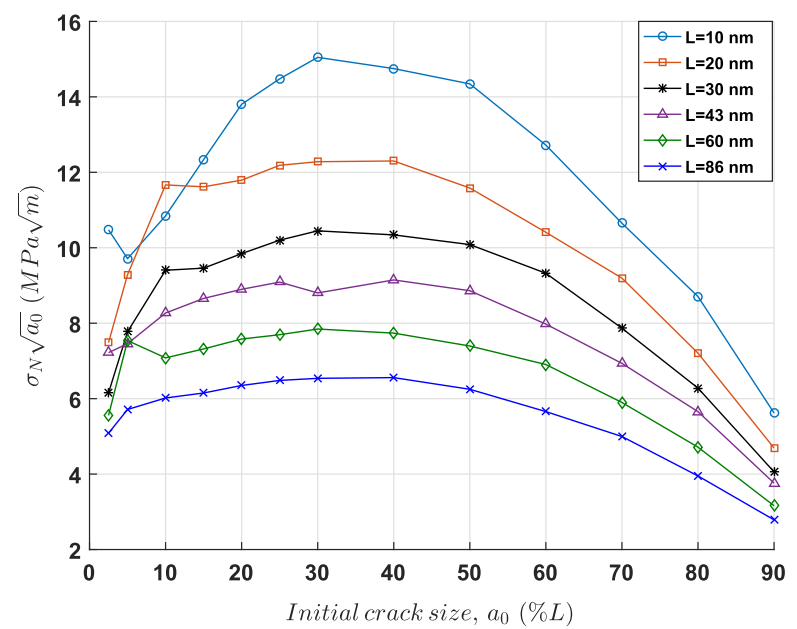

(c) Lattice oriented along $30^{\circ}$

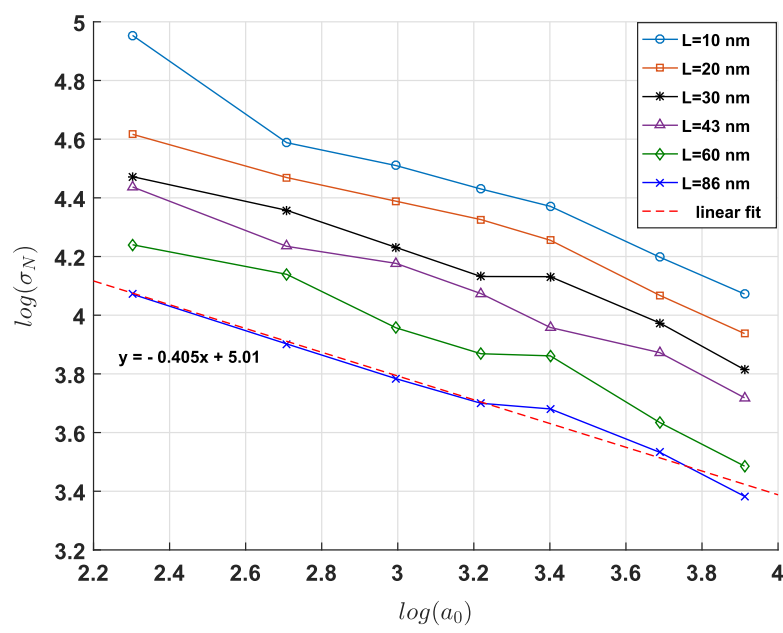

(e) Lattice oriented along $13.9^{\circ}$

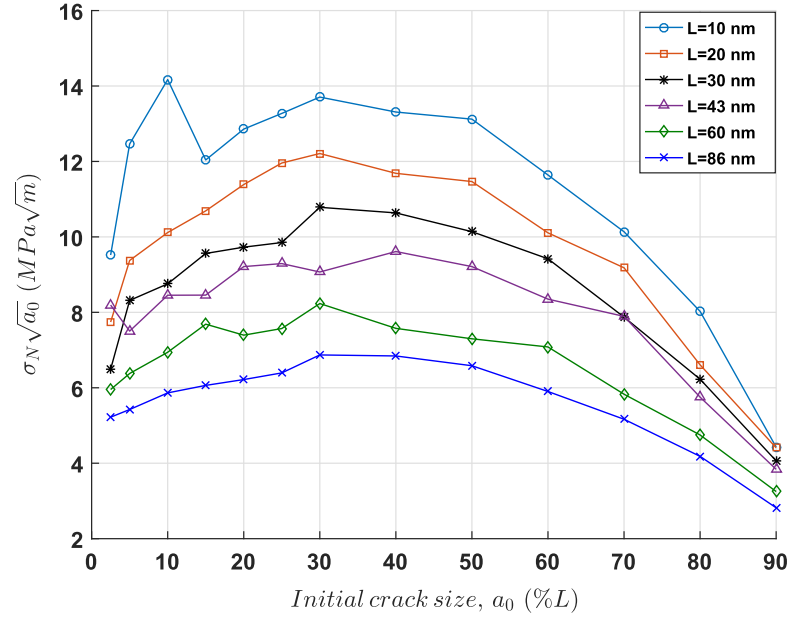

(b) Lattice oriented along $13.9^{\circ}$

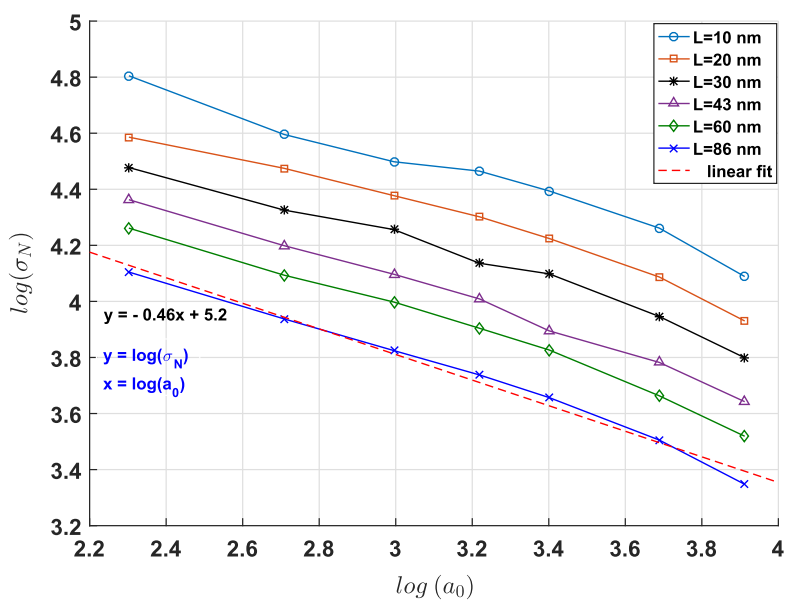

(d) Lattice oriented along $0^{\circ}$

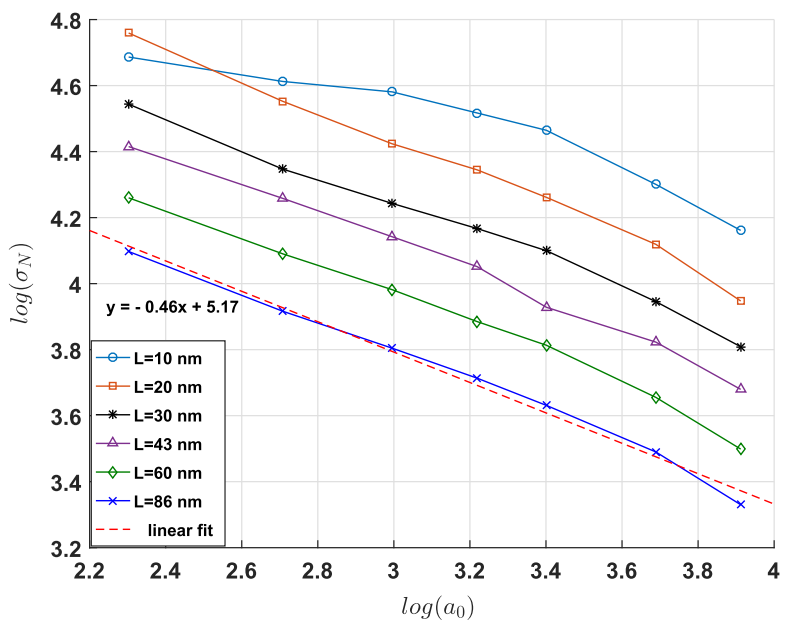

(f) Lattice oriented along $30^{\circ}$

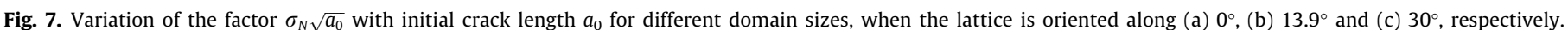

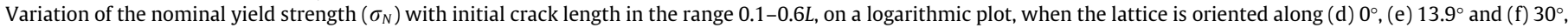

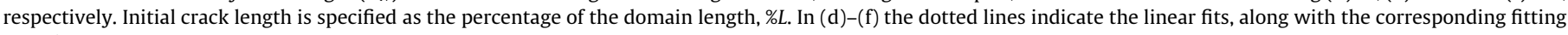
equation.

product of $\sigma_{N} \sqrt{a_{0}}$. Fig. 7(a)-(c) shows the variation of the factor $\sigma_{N} \sqrt{a_{0}}$ with initial crack length $a_{0}$ for different domain sizes, when the lattice is oriented along the $0^{\circ}, 13.9^{\circ}$ and $30^{\circ}$, respectively. Initial crack length is specified as the percentage of the domain length,
$\% L$. Values of the yield stress and the factor $\sigma_{N} \sqrt{a_{0}}$ for initial crack lengths between $0.1 \mathrm{~L}$ and $0.6 \mathrm{~L}$ are listed in Table 1 , for different domain sizes, when the lattice is oriented along the $0^{\circ}$. The mean and standard deviation of the factor $\sigma_{N} \sqrt{a_{0}}$ in Table 1 , and for 
Table 1

Distribution of the nominal yield strength with crack and domain sizes, when the lattice is oriented along the $0^{\circ}$ direction.

\begin{tabular}{|c|c|c|c|c|c|c|c|c|c|}
\hline \multirow[t]{2}{*}{ Sample No. } & \multirow[t]{2}{*}{ Crack length $a_{0}(\% L)$} & \multicolumn{2}{|c|}{$\sigma_{N}(\mathrm{GPa})$} & \multicolumn{6}{|c|}{ Factor $\sigma_{N} \sqrt{a_{0}}(\mathrm{MPa} \sqrt{\mathrm{m}})$ with domain size, $L(\mathrm{~nm})$} \\
\hline & & $L=10$ & $L=43$ & 10 & 20 & 30 & 43 & 60 & 86 \\
\hline 1 & 10.0 & 122.098 & 78.482 & 12.210 & 9.810 & 8.802 & 7.848 & 7.091 & 6.062 \\
\hline 2 & 15.0 & 99.049 & 66.613 & 12.130 & 10.751 & 9.262 & 8.158 & 7.343 & 6.283 \\
\hline 3 & 20.0 & 89.848 & 60.136 & 12.710 & 11.258 & 9.974 & 8.504 & 7.702 & 6.477 \\
\hline 4 & 25.0 & 86.900 & 55.088 & 13.740 & 11.674 & 9.891 & 8.710 & 7.844 & 6.641 \\
\hline 5 & 30.0 & 80.960 & 49.150 & 14.022 & 11.835 & 10.433 & 8.513 & 7.951 & 6.710 \\
\hline 6 & 40.0 & 70.916 & 43.919 & 14.183 & 11.913 & 10.348 & 8.784 & 7.797 & 6.658 \\
\hline 7 & 50.0 & 59.661 & 38.179 & 13.340 & 11.386 & 9.985 & 8.537 & 7.547 & 6.358 \\
\hline 8 & 60.0 & 49.535 & 31.895 & 12.133 & 10.164 & 9.165 & 7.812 & 6.894 & 5.788 \\
\hline
\end{tabular}

Table 2

Mean and standard deviation of the factor $\sigma_{N} \sqrt{a_{0}}$ in Table 1 , for different domain sizes with lattice oriented along $0^{\circ}, 13.9^{\circ}$ and $30^{\circ}$.

\begin{tabular}{|c|c|c|c|c|c|c|}
\hline Angle & 10 & 20 & 30 & 43 & 60 & 86 \\
\hline \multicolumn{7}{|c|}{ Mean of the factor $\sigma_{N} \sqrt{a_{0}}$ with domain size } \\
\hline $0^{\circ}$ & 13.058 & 11.099 & 9.732 & 8.358 & 7.521 & 6.372 \\
\hline $13.9^{\circ}$ & 13.014 & 11.202 & 9.858 & 8.957 & 7.472 & 6.342 \\
\hline $30^{\circ}$ & 13.541 & 11.728 & 9.880 & 8.712 & 7.444 & 6.250 \\
\hline \multicolumn{7}{|c|}{ Standard deviation of the factor $\sigma_{N} \sqrt{a_{0}}$ with domain size } \\
\hline $0^{\circ}$ & 0.867 & 0.783 & 0.589 & 0.374 & 0.379 & 0.322 \\
\hline $13.9^{\circ}$ & 0.831 & 0.808 & 0.661 & 0.472 & 0.399 & 0.397 \\
\hline $30^{\circ}$ & 1.449 & 0.612 & 0.446 & 0.403 & 0.332 & 0.306 \\
\hline
\end{tabular}

different domain sizes with lattice oriented along $0^{\circ}, 13.9^{\circ}$ and $30^{\circ}$, are shown in Table 2.

From Table 2, the maximum and minimum standard deviations are observed to be 1.449 and 0.306 , respectively. Furthermore, variation of the yield strength with initial crack lengths in the range $0.1-0.6 L$ are plotted in Fig. $7(\mathrm{~d})-(\mathrm{f})$, when the lattice is oriented along the $0^{\circ}, 13.9^{\circ}$ and $30^{\circ}$, respectively. The dotted lines in Fig. 7(d)-(f) indicate the linear fits and the corresponding fitted equations for the domain size $86 \times 86 \mathrm{~nm}^{2}$. From the fitted equations the slope of the curve is observed to be $-0.46,-0.405$ and -0.46 , when the lattice is oriented along the $0^{\circ}, 13.9^{\circ}$ and $30^{\circ}$, respectively. Also the slopes are observed to gradually increase with the domain size, approaching -0.5 in the limit of infinite domain size, $60 \mathrm{~nm}$ in present work. Therefore, based on Fig. 7, Tables 1 and 2, $\sigma_{N}$ can be assumed to vary as $1 / \sqrt{a_{0}}$ and hence the factor $\sigma_{N} \sqrt{a_{0}}$ can be assumed to be constant for initial crack lengths between $0.1 \mathrm{~L}-0.6 \mathrm{~L}$. Furthermore, the curves in Fig. 7(a)(c) tend to become straight as the domain size increases, indicating that the deviation with the mean reduces as the domain size increases. This is further confirmed by the gradual reduction of the standard deviation values with the domain size as listed in Table 2. This behavior is expected and is in agreement with the continuum theory [42]. However, for small domain sizes and small and large initial crack lengths, the continuum theory fails [40]. In this paper, $a_{0} \leqslant 0.1 L$ and $a_{0} \geqslant 0.6 L$ are considered as small and large initial crack lengths, respectively.

\section{Conclusions}

Molecular dynamics based simulations have been carried out to investigate the combined effect of domain size, crack length and lattice orientation on the yield properties of Graphene. Six different square domains with three different lattice orientations and thirteen different initial crack lengths are considered to carry out a total of 252 simulations. Results from molecular dynamics simulations are correlated to the continuum based size-effect law and the multifractal scaling law. The yield strength is observed to vary as $\approx L^{-1 / 3}$. This is in agreement with MFSL. Furthermore, the yield strength is observed to vary as the inverse square of the initial crack length $\approx a_{0}^{-1 / 2}$, when the initial crack lengths lie in the range $0.1-0.6 L$, in agreement with the Griffith's theory of fracture.

Distribution of the stress-strain plots with varying domain sizes, initial crack lengths and lattice orientations are studied. The yield values are observed to drop significantly for small initial crack lengths less than or equal to $0.1 \mathrm{~L}$. The yield values are also observed to decrease with the increase in domain size, as the larger specimens offer more spaces for dislocations to initiate. Combined effect of the domain size, lattice orientation and crack length on the yield values of stress and strain have also been put into evidence.

\section{Acknowledgements}

Javvaji and Roy Mahapatra thankfully acknowledge the use of computational facilities at the ACECOST Computational Science Lab, Department of Aerospace Engineering, IISc and funding under ACECOST Phase-III program of Aeronautics Research and Development Board, India. Paggi and Budarapu acknowledge funding from the European Research Council (ERC, Belgium), Grant No. 306622 through the ERC Starting Grant "Multi-field and multi-scale Computational Approach to Design and Durability of PhotoVoltaic Modules" - CA2PVM. Zi appreciates the financial support through Grant No. 20133010021770, from the New \& Renewable Energy Core Technology Program of the Korea Institute of Energy Technology Evaluation and Planning (KETEP), Ministry of Trade, Industry \& Energy, Republic of Korea.

\section{References}

[1] Z. Bažant, Size effect in blunt fracture: concrete, rock, metal, J. Eng. Mech. ASCE 10 (1984) 518-535, http://dx.doi.org/10.1061/(ASCE)0733-9399(1984)110:4 (518).

[2] Z. Bažant, Size effect aspects of measurement of fracture characteristics of quasi-brittle material, Adv. Cement Mater. 4 (1996) 128-137, http://dx.doi. org/10.1016/S1065-7355(96)00037-5.

[3] Z. Bažant, E.-P. Chen, Scaling of structural failure, Appl. Mech. Rev. 50 (1997) 593-627, http://dx.doi.org/10.2172/420364.

[4] Z. Bažant, I. Daniel, Z. Li, Size effect and fracture characteristics of composite laminates, J. Eng. Mater. Technol. 118 (1996) 317-324, http://dx.doi.org/ 10.1115/1.2806812.

[5] Z. Bažant, S.-G. Lee, P. Pfeiffer, Size effect tests and fracture characteristics of aluminum, Eng. Fract. Mech. 1 (1987) 45-57, http://dx.doi.org/10.1016/00137944(87)90078-6.

[6] Z. Bažant, Y. Xi, Statistical size effect in quasi-brittle structures. II: Nonlocal theory, J. Eng. Mech. 117 (1991) 2623-2640, http://dx.doi.org/10.1061/(ASCE) 0733-9399(1991)117:11(2623).

[7] Z. Bažant, A. Yavari, Response to A. Carpinteri, B. Chiaia, P. Cornetti and S. Puzzi's comments on "is the cause of size effect on structural strength fractal or energetic-statistical?”, Eng. Fract. Mech. 74 (2007) 2897-2910, http://dx. doi.org/10.1016/j.engfracmech.2009.12.005.

[8] Z. Bažant, Q. Yu, Universal size effect law and effect of crack depth on quasibrittle structure strength, J. Eng. Mech. 135 (2) (2009) 78-84, http://dx.doi.org 10.1061/(ASCE)0733-9399(2009)135:2(78).

[9] H. Bu, Y. Chen, M. Zou, H. Yi, K. Bi, Z. Ni, Atomistic simulations of mechanical properties of graphene nanoribbons, Phys. Lett. A 373 (2009) 3359-3362, http://dx.doi.org/10.1016/j.physleta.2009.07.048.

[10] P. Budarapu, B. Javvaji, V. Sutrakar, D. Mahapatra, G. Zi, T. Rabczuk, Crack propagation in graphene, J. Appl. Phys. 118 (2015) 382-395, http://dx.doi.org/ $10.1063 / 1.4928316$ 
[11] P. Budarapu, Y. Sudhir, J. Brahmanandam, D. Mahapatra, Vibration analysis of multi-walled carbon nanotubes embedded in elastic medium, Front. Struct. Civil Eng. 8 (2) (2014) 151-159, http://dx.doi.org/10.1007/s11709-014-0247-9.

[12] A. Carpinteri, Fractal nature of material microstructure and size effects on apparent mechanical properties, Mech. Mater. 18 (1993) 89-101, http://dx. doi.org/10.1016/0167-6636(94)00008-5.

[13] A. Carpinteri, Scaling laws and renormalization groups for strength and toughness of disordered materials, Int. J. Solids Struct. 31 (3) (1994) 291-302, http://dx.doi.org/10.1016/0020-7683(94)90107-4.

[14] A. Carpinteri, B. Chiaia, P. Cornetti, A scale-invariant cohesive crack model for quasi-brittle materials, Eng. Fract. Mech. 69 (2002) 207-217, http://dx.doi.org/ 10.1016/S0013-7944(01)00085-6.

[15] A. Carpinteri, B. Chiaia, G. Ferro, Size defects on normal tensile strength of concrete structures: multifractality of material ligaments and dimensional transition from order to disorder, Mater. Struct. 28 (1995) 311-317, http://dx. doi.org/10.1007/BF02473145.

[16] A. Carpinteri, P. Cornetti, A fractional calculus approach to the description of stress and strain localization in fractal media, Chaos Solitons Fractals 13 (2002) 85-94, http://dx.doi.org/10.1016/S0960-0779(00)00238-1.

[17] A. Carpinteri, P. Cornetti, S. Puzzi, Scaling laws and multi-scale approach to the mechanics of heterogeneous and disordered materials, Appl. Mech. Rev. 59 (2006) 283-305, http://dx.doi.org/10.1115/1.2204076.

[18] A. Carpinteri, S. Puzzi, A fractal approach to indentation size effect, Eng. Fract Mech. $73 \quad$ (2006) 2110-2122, http://dx.doi.org/10.1016/j. engfracmech.2006.04.020.

[19] K. Chen, S. Bin, Y. Yonghai, Q. Juanjuan, G. Lin, Binary synergy strengthening and toughening of bio-inspired nacre-like graphene oxide/sodium alginate composite paper, ACS Nano 9 (8) (2015) 8165-8175, http://dx.doi.org/ 10.1021/acsnano.5b02333.

[20] Y. Chu, T. Ragab, C. Basaran, The size effect in mechanical properties of finitesized graphene nanoribbon, Comput. Mater. Sci. 81 (2014) 269-274, http://dx. doi.org/10.1016/j.commatsci.2013.08.016.

[21] D. Datta, S. Nadimpalli, L. Yinfeng, V. Shenoy, Effect of crack length and orientation on the mixed-mode fracture behavior of graphene, Extreme Mech. Lett. 5 (2015) 10-17, http://dx.doi.org/10.1016/j.eml.2015.08.

[22] M. Dewapriya, R. Rajapakse, Molecular dynamics simulations and continuum modeling of temperature and strain rate dependent fracture strength of graphene with vacancy defects, J. Appl. Mech. 81 (8) (2014) 081010.

[23] M. Dewapriya, R. Rajapakse, A. Phani, Atomistic and continuum modelling of temperature-dependent fracture of graphene, Int. J. Fract. 187 (2014) 199212.

[24] R. Faccio, P. Denis, H. Pardo, C. Goyenola, A. Mombr, Mechanical properties of graphene nanoribbons, J. Phys.: Condens. Matter 21 (2009) 285304, http://dx. doi.org/10.1088/0953-8984/21/28/285304.

[25] A. Gamboa, G. Vignoles, J. Leyssale, On the prediction of graphene's elastic properties with reactive empirical bond order potentials, Carbon 89 (2015) 176-187, http://dx.doi.org/10.1016/j.carbon.2015.03.035.

[26] A. Geim, Graphene: status and prospects, Science 324 (2009) 1530-1534 http://dx.doi.org/10.1126/science.1158877.

[27] S. Georgantzinos, G. Giannopoulos, D. Katsareas, P. Kakavas, N. Anifantis, Sizedependent non-linear mechanical properties of graphene nanoribbons, Comput. Mater. Sci. 50 (2011) 2057-2062, http://dx.doi.org/10.1016/ j.commatsci.2011.02.008.

[28] R. Grantab, V. Shenoy, R. Ruoff, Anomalous strength characteristics of tilt grain boundaries in graphene, Science 330 (2010) 946-948, http://dx.doi.org $10.1126 /$ science.1196893.

[29] A. Griffith, The phenomena of rapture and flow in solids, Philos. Trans. Roy Soc. London A 221 (1921) 163-198, http://dx.doi.org/10.1098/rsta.1921.0006.

[30] C. Jin, H. Lan, L. Peng, K. Suenaga, S. Iijima, Deriving carbon atomic chains from graphene, Phys. Rev. Lett. 102 (2009) 205501, http://dx.doi.org/10.1103/ PhysRevLett.102.205501.

[31] K. Kim, V. Artyukhov, W. Regan, Y. Liu, M. Crommie, B. Yakobson, A. Zettl, Ripping graphene: preferred directions, Nano Lett. 12 (2012) 293-297, http:// dx.doi.org/10.1021/nl203547z.

[32] Y. Kim, J. Lee, M. Yeom, J. Shin, H. Kim, Y. Cui, J. Kysar, J. Hone, Y. Jung, S. Jeon, S Han, Strengthening effect of single-atomic-layer graphene in metal-graphene nanolayered composites, Nat. Commun. 4 (2013) 2114, http://dx.doi.org $10.1038 /$ ncomms3114.

[33] K. Kostarelos, K. Novoselov, Graphene devices for life, Nat. Nanotechnol. 9 (2014) 744-745, http://dx.doi.org/10.1038/nnano.2014.224.

[34] C. Lee, W. Xiaoding, W.K. Jeffrey, H. James, Measurement of the elastic properties and intrinsic strength of monolayer graphene, Science 321 (5887) (2008) 385-388, http://dx.doi.org/10.1126/science.1157996.

[35] F. Liu, P. Ming, J. Li, Ab initio calculation of ideal strength and phonon instability of graphene under tension, Phys. Rev. B 76 (2007) 064120, http:// dx.doi.org/10.1103/PhysRevB.76.064120.

[36] Y. Liu, B. Xie, Z. Zhang, Q. Zheng, Z. Xu, Mechanical properties of graphene papers, J. Mech. Phys. Solids 60 (2012) 591-605, http://dx.doi.org/10.1016/j. jmps.2012.01.002.

[37] Q. Lu, W. Gao, R. Huang, Atomistic simulation and continuum modeling of graphene nanoribbons under uniaxial tension, Modell. Simul. Mater. Sci. Eng. 19 (2011) 54006, http://dx.doi.org/10.1088/0965-0393/19/5/054006.

[38] G. Marc, W. McMillan, The virial theorem, Adv. Chem. Phys. 58 (1985) 209, http://dx.doi.org/10.1002/9780470142820.

[39] A. Morpurgo, Ten years of nature physics: the ABC of 2D materials, Nat. Phys. 11 (2015) 625-626, http://dx.doi.org/10.1038/nphys3516.
[40] A. Orlov, I. Ovid'ko, Mechanical properties of graphene nanoribbons: a selective review of computer simulations, Rev. Adv. Mater. Sci. 40 (2015) 249-256.

[41] M. Paggi, A. Carpinteri, Fractal and multifractal approaches for the analysis of crack-size dependent scaling laws in fatigue, Chaos Solitons Fractals 40 (2009) 1136-1145, http://dx.doi.org/10.1016/j.ijfatigue.2015.09.009.

[42] Z. Peng, M. Lulu, F. Feifei, Z. Zhi, P. Cheng, L. Phillip E, L. Zheng, G. Yongji, Z. Jiangnan, Z. Xingxiang, A. Pulickel M, Z. Ting, L. Jun, Fracture toughness of graphene, Nat. Commun. 5 (2014) 3782, http://dx.doi.org/10.1038/ ncomms4782 (2014).

[43] S. Plimpton, Fast parallel algorithms for short-range molecular dynamics, J. Comput. Phys. 117 (1995) 1-19, http://dx.doi.org/10.1006/jcph.1995.1039.

[44] O. Shenderova, D. Brenner, A. Omeltchenko, X. Su, L. Yang, Atomistic modeling of the fracture of polycrystalline diamond, Phys. Rev. B 61 (6) (2000) 38773888, http://dx.doi.org/10.1103/PhysRevB.61.3877.

[45] K. Spanos, S. Georgantzinos, N. Anifantis, Mechanical properties of graphene nanocomposites: a multiscale finite element prediction, Compos. Struct. (132) (2015), http://dx.doi.org/10.1016/j.compstruct.2015.05.078.

[46] A. Subramaniyan, C. Sun, Continuum interpretation of virial stress in molecular simulations, Int. J. Solids Struct. 45 (2008) 4340-4346, http://dx.doi.org/ 10.1016/j.ijsolstr.2008.03.016.

[47] Y. Sun, F. Ma, K. Xu, Size dependent mechanical properties of graphene nanoribbons: molecular dynamics simulation, Mater. Sci. Forum 749 (2013) 456-460, http://dx.doi.org/10.4028/www.scientific.net/MSF.749.456.

[48] W. Swope, H. Anderson, P. Berens, K. Wilson, A computer simulation method for the calculation of equilibrium constants for the formation of physical clusters of molecules: application to small water clusters, J. Chem. Phys. 76 (1982) 637, http://dx.doi.org/10.1063/1.442716.

[49] J. Tersoff, Modeling solid-state chemistry: Interatomic potentials for multicomponent systems, Phys. Rev. B 39 (8) (1989) 5566-5568, http://dx. doi.org/10.1103/PhysRevB.39.5566.

[50] S. Thomas, K. Ajith, Molecular dynamics simulation of the thermo-mechanical properties of monolayer graphene sheet, Procedia Mater. Sci. 5 (2014) 489498, http://dx.doi.org/10.1016/j.mspro.2014.07.292.

[51] C. Tiwary, B. Javvaji, C. Kumar, D. Mahapatra, S. Ozden, P. Ajayan, K. Chattopadhyay, Chemical-free graphene by unzipping carbon nanotubes using cryo-milling, Carbon (2015) 217-224, http://dx.doi.org/10.1016/ j.carbon.2015.03.036.

[52] K.Y. Volokh, On the strength of graphene, J. Appl. Mech. 79 (6) (2012) 064501, http://dx.doi.org/10.1115/1.4005582.

[53] S. Wang, J. Guo, Y. Jiang, The size- and chirality-dependent elastic properties of graphene nanofilms, J. Comput. Theor. Nanosci. 10 (2013) 250-256, http://dx. doi.org/10.1166/jctn.2013.2687.

[54] C. Xu, T. Xue, J. Guo, Y. Kang, W. Qiu, H. Song, H. Xie, An experimental investigation on the tangential interfacial properties of graphene: size effect Mater. Lett. 161 (2015) 755-758, http://dx.doi.org/10.1016/ j.matlet.2015.09.088.

[55] Z. Yang, Y. Huang, F. Maa, Y. Sune, K. Xu, P. Chu, Size-dependent deformation behavior of nanocrystalline graphene sheets, Mater. Sci. Eng. B 198 (2015) 95101, http://dx.doi.org/10.1016/j.mseb.2015.03.019.

[56] H. Yin, H. Qi, F. Fan, T. Zhu, B. Wang, Y. Wei, Griffith criterion for brittle fracture in graphene, Nano Lett. 15 (2015) 1918-1924.

[57] X. Yu, Y. Shen, T. Liu, W. Tao(Tom), Q. Wang, Photocurrent generation in lateral graphene p-n junction created by electron-beam irradiation, Sci. Rep. 5 (2015) 12014, http://dx.doi.org/10.1038/srep12014.

[58] T. Zhang, X. Li, H. Gao, Fracture of graphene: a review, Int. J. Fract. (2015) 1-31, http://dx.doi.org/10.1007/s10704-015-0039-9.

[59] H. Zhao, N. Aluru, Temperature and strain-rate dependent fracture strength of graphene, J. Appl. Phys. 108 (2010) 064321, http://dx.doi.org/10.1088/00223727/47/42/425301.

[60] H. Zhao, K. Min, N. Aluru, Size and chirality dependent elastic properties of graphene nanoribbons under uniaxial tension, Nano Lett. 9 (8) (2009) 30123015, http://dx.doi.org/10.1021/n1901448z.

[61] W. Zhao, W. Yanlei, W. Zhangting, W. Wenhui, B. Kedong, L. Zheng, Y. Juekuan, C. Yunfei, X. Zhiping, N. Zhenhua, Defect-engineered heat transport in graphene: a route to high efficient thermal rectification, Sci. Rep. 5 (2015) 11962, http://dx.doi.org/10.1038/srep11962.

[62] Y. Zheng, L. Xu, Z. Fan, N. Wei, Z. Huang, A molecular dynamics investigation of the mechanical properties of graphene nanochains, J. Mater. Chem. 22 (2012) 9798-9805, http://dx.doi.org/10.1039/C2JM16626G.

[63] P. Budarapu, R. Gracie, S. Bordas, T. Rabczuk, An adaptive multiscale method for quasi-static crack growth, Comput. Mech. 53 (6) (2014) 1129-1148.

[64] P. Budarapu, R. Gracie, Y. Shih-Wei, X. Zhuang, T. Rabczuk, Efficient Coarse Graining in Multiscale Modeling of Fracture, Theor. Appl. Fract. Mec. 69 (2014) 126-143.

[65] H. Talebi, M. Silani, T. Rabczuk, Concurrent Multiscale Modelling of Three Dimensional Crack and Dislocation Propagation, Adv. Eng. Softw. 80 (2015) 82-92.

[66] H. Talebi, M. Silani, S. Bordas, P. Kerfriden, T. Rabczuk, A Computational Library for Multiscale Modelling of Material Failure, Comput. Mech. 53 (5) (2014) 1047-1071.

[67] H. Talebi, M. Silani, S.P.A. Bordas, P. Kerfriden, T. Rabczuk, Molecular Dynamics/ XFEM Coupling by a Three-Dimensional Extended Bridging Domain with Applications to Dynamic Brittle Fracture, Int. J. Mult. Comp. Eng. 11 (6) (2013) 527-541.

[68] P.R. Budarapu, B. Javvaji, V.K. Sutrakar, D. Roy Mahapatra, M. Paggi, G. Zi, T. Rabczuk, Lattice orientation and crack size effect on the mechanical properties of Graphene, Int. J. Fract. http://dx.doi.org/10.1007/s10704-016-0115-9. 